\title{
Transmettre le patrimoine comme un tout - une initiative dans le Parc naturel régional des Caps et Marais d'Opale
}

Passing on the heritage as a whole - an experimentation from Caps et Marais d'Opale Regional nature Park

\section{Delphine Panossian}

\section{(Q) OpenEdition}

\section{Journals}

Édition électronique

URL : http://journals.openedition.org/developpementdurable/13817

DOI : 10.4000/developpementdurable.13817

ISSN : 1772-9971

Éditeur

Association DD\&T

Référence électronique

Delphine Panossian, «Transmettre le patrimoine comme un tout - une initiative dans le Parc nature régional des Caps et Marais d'Opale», Développement durable et territoires [En ligne], Vol. 10, $n^{\circ} 1 \mid$ Avril 2019, mis en ligne le 04 avril 2019, consulté le 30 avril 2019. URL : http://journals.openedition.org/ developpementdurable/13817 ; DOI : 10.4000/developpementdurable.13817

Ce document a été généré automatiquement le 30 avril 2019

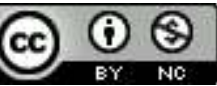

Développement Durable et Territoires est mis à disposition selon les termes de la licence Creative Commons Attribution - Pas d'Utilisation Commerciale 4.0 International. 


\section{Transmettre le patrimoine comme un tout - une initiative dans le Parc naturel régional des Caps et Marais d'Opale}

Passing on the heritage as a whole - an experimentation from Caps et Marais

d'Opale Regional nature Park

Delphine Panossian

1 Le Parc naturel régional des Caps et Marais d'Opale (Carte 1) est le fruit de la fusion de deux parcs bien ancrés dans les territoires du Boulonnais et de l'Audomarois, survenue en 2000. 
Carte 1. Le Parc naturel régional des Caps et Marais d'Opale

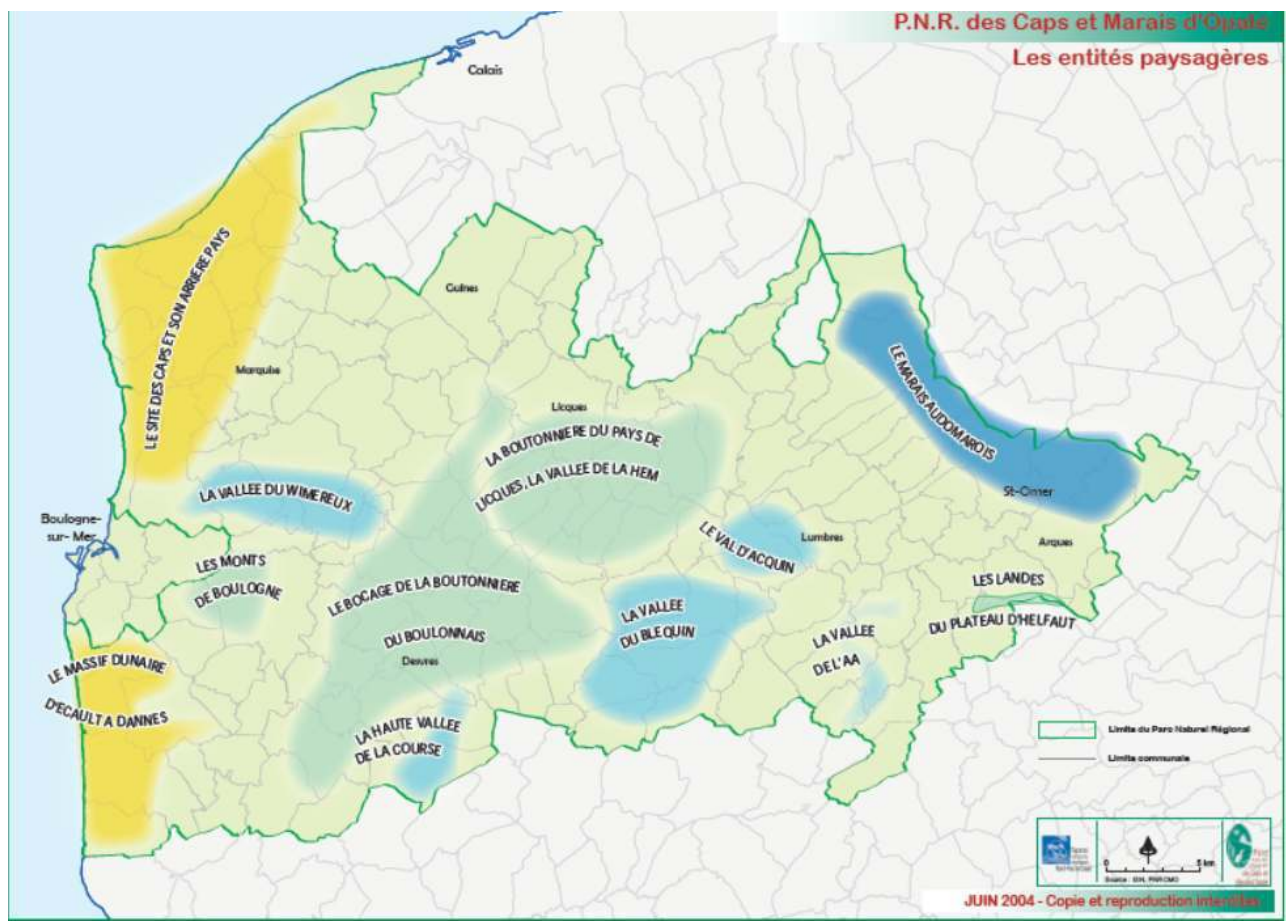

Source : PNRCMO

2 Selon la Fédération des Parcs naturels régionaux, « peut être classé Parc naturel régional un territoire à dominante rurale dont les paysages, les milieux naturels et le patrimoine culturel sont de grande qualité, mais dont l'équilibre est fragile. C'est la préservation des richesses naturelles, culturelles et humaines (traditions populaires, savoir-faire techniques) qui est à la base du projet de développement des Parcs naturels régionaux ${ }^{1}{ }$. Le parc des Caps et Marais d'Opale s'inscrit dans cette philosophie : il s'agit de travailler pour un territoire équilibré, liant préservation des patrimoines et économie, autrement dit, en reliant l'environnement aux activités humaines. C'est en s'appuyant sur cette ambition que le Parc a travaillé des programmes autour des relations que les habitants entretiennent avec leur environnement, dans un territoire où la nature est fortement anthropisée.

Dans les années 1990, les travaux des parcs de l'Audomarois et du Boulonnais avaient l'habitude d'intégrer une dimension ethnologique et historique. Quelques années plus tard, en 2005, il est fait le constat que la dimension humaine est moins étudiée et utilisée, au profit d'actions thématisées et moins transversales. À cette époque, c'est la connaissance et la préservation des espaces naturels qui prime, sans forcément associer les habitants, hormis les écoliers, dans ces programmes. Ces actions présenteront des résultats scientifiques certains, mais pourront parfois contribuer à rendre le territoire plus complexe à appréhender pour les habitants. C'est pourquoi, au milieu d'autres actions éducatives, un programme de mobilisation des habitants par la pratique artistique voit le jour. Son objectif est de les amener à se rencontrer autour d'une création collective participative. C'est au fur et à mesure de ces projets artistiques que la prise en compte du territoire comme un tout a su montrer son intérêt pour convaincre que les patrimoines qui constituent le territoire du parc forment un bien commun. 


\section{La nécessité de faire des "pas de côté »}

4 La spécificité des parcs est de considérer les patrimoines culturel, naturel et paysager au même niveau. Le label Parc n'implique aucune protection supplémentaire et, pour autant, les objectifs de transmission des patrimoines sont ambitieux. Les modes de réflexion et de travail qui en découlent sont forcément très différents de ceux qui peuvent être mis en œuvre ailleurs. Si le parc doit préserver le patrimoine naturel, il n'en est pas l'acteur principal. Le parc doit donc rechercher la conviction et la motivation des acteurs locaux, et notamment des habitants, pour les impliquer dans le projet du territoire. La place de l'habitant y est centrale.

5 Le profil de l'habitant du parc s'est modifié au fil du temps. Si, au moment de la création des parcs, dans les années 1960, les habitants étaient principalement agriculteurs ou ouvriers, aujourd'hui, la population est plus hétérogène. Des cadres ou des professions intermédiaires viennent habiter dans les parcs soit par choix (le « retour à la terre »), soit par nécessité (le prix du foncier en ville étant trop élevé). Ces mouvements que connait le territoire depuis les années 1970 ont donné naissance à certains conflits, expliqués en partie par des perceptions assez tranchées sur ce que, par exemple, doit être l'agriculture. En parallèle, certaines évolutions de l'espace rural sont à noter. La première qui a modifié le rapport qu'entretiennent les paysans avec la terre, leur outil de travail, est l'arrivée du moteur qui a engagé l'agriculture vers un mode de faire plus productiviste. Pour sortir de la composante uniquement agricole, la société s'individualise; là où les campagnes vivaient autour des cafés de village, ceux-ci ferment un à un. Le lien social s'y trouve abîmé, avec de nouveaux arrivants qui participent peu à la vie du village et connaissent peu le territoire. De surcroit, l'explosion des nouvelles technologies a contribué à surinformer les habitants sur les problématiques globales du changement climatique ou des scandales sanitaires. En revanche, ces mêmes habitants font très peu le lien avec ce qu'il se passe chez eux ou dans leur village et s'engagent faiblement au niveau local.

6 Ces quelques tendances n'ont pas vocation à caricaturer ce qu'est l'espace rural aujourd'hui, mais à apporter un éclairage pour expliquer l'action du parc. L'habitant du parc occupe de plus en plus un lieu sans le « vivre ». Il l'occupe, mais n'en partage que peu le quotidien, l'histoire, les valeurs. Ainsi, pour mobiliser autour de la préservation de la nature, ou plus généralement de l'environnement, il a été nécessaire de penser des projets différemment, en s'autorisant quelques « pas de côté ».

\section{Toucher autrement}

\subsection{Développer la pratique culturelle}

7 Pour répondre au premier objectif d'impliquer les habitants dans le projet du territoire, le parc a poursuivi des expériences afin d'intéresser un public plus large. En effet, dans les années 2000, le parc avait développé un programme ambitieux d'éducation au territoire destiné aux écoles. Cette action a rencontré un vif succès. Sa seule limite était de s'adresser exclusivement au public scolaire. Ainsi, pour élargir son audience, le parc a mis au point un programme de développement de la pratique artistique intitulé «Dans le sillon des artistes»(Panossian, 2008). Le parc faisait le pari alors que des habitants amenés à s'impliquer dans une création artistique collective pourraient le faire dans 
d'autres domaines. Le «sillon» avait été imaginé comme un "premier pas». Ce programme a débuté avec six actions artistiques de courte durée se terminant par une restitution publique, ouverte à tous les habitants du village. Plusieurs projets ont été menés : une création musicale évoquant la mare et intégrant des jeunes d'un centre de loisirs (figure 1); la réalisation d'une exposition photo de portraits d'habitants d'une commune rurale...

Figure 1. Mare en musique à Ecault

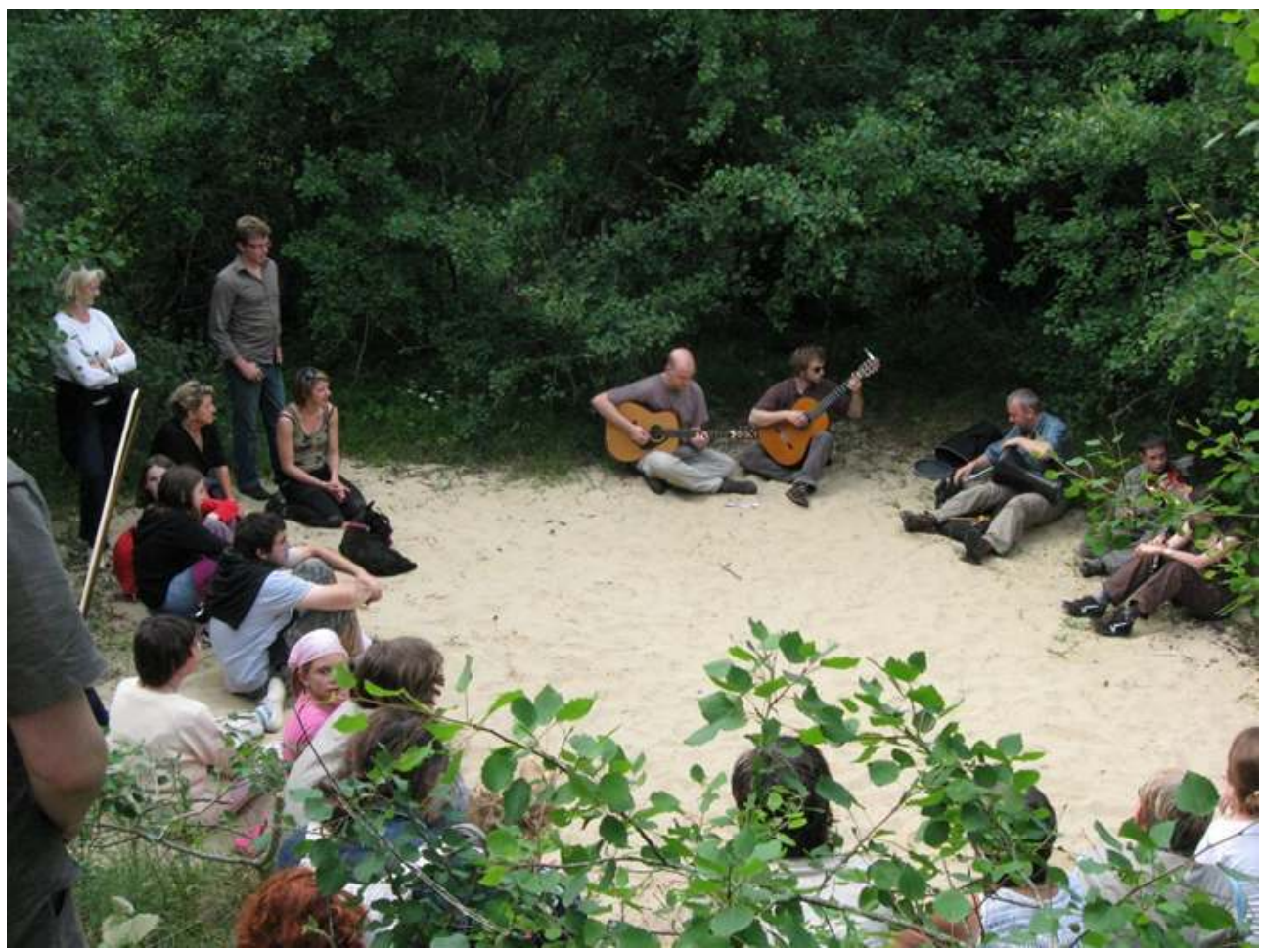

Source : PNRCMO

Cette première salve d'actions culturelles a permis au parc d'éprouver cette nouvelle forme d'implication citoyenne.

9 L'une des conclusions, plutôt encourageante, s'entendait dans les propos des élus au moment des restitutions : « On rencontre des gens que nous n'avons pas l'habitude de voir. » $\mathrm{Ce}$ programme a pu, à sa manière, apporter le terreau pour dynamiser le lien social dans les communes.

\subsection{Renouer avec son territoire}

10 Cette action a très vite été recentrée. Il a été proposé qu'elle vienne appuyer un programme en cours. Pensé en transversalité, le «Sillon des artistes » devait revêtir un nouvel objectif : celui d'emmener les habitants vers la compréhension et la préservation du territoire. Ainsi, de premières actions se sont déroulées avec comme thème central la nature. Au-delà de la participation, il fallait que ces actions puissent permettre aux habitants de tirer des enseignements.

11 C'est ainsi que la restitution publique d'un spectacle créé avec des habitants de communes de la vallée de la Hem a accueilli une bonne centaine de curieux qui savaient 
que le dernier berger du village allait monter sur scène pour se raconter... et raconter le pays et ses évolutions, en quelque sorte. Le seul fait d'intégrer des personnes considérées comme «sachants» locaux (Carré, 2007) ${ }^{2}$ a profité à la mobilisation des habitants. Le témoignage d'un berger, encore en activité dans les années 1990 est plein d'enseignements et permet un recul sur notre rapport à la nature. Ce berger était en contact quotidien avec ses "monts", comme ses moutons. Utiliser son témoignage, c'était permettre aux habitants, notamment ceux non originaires du "coin", de se familiariser avec cette nature calcicole toute proche. Le témoignage du berger, mis en valeur par le spectacle, est donc devenu à lui seul un moyen efficace d'intéresser à la préservation de ces coteaux. Ce spectacle a constitué la première action de valorisation qui venait appuyer un plan d'action centré principalement sur les enjeux écologiques. Cette expérience a conforté le parc dans l'idée que lorsque l'on parle de nature actuelle, nous ne sommes jamais loin des pratiques anciennes et des histoires avec un grand et des petits $\mathrm{H}$.

Figure 2. L'ancien berger du village, invité sur scène à se raconter, le soir de la restitution du spectacle Où s'en vont ces gais bergers - 2007, Audrehem

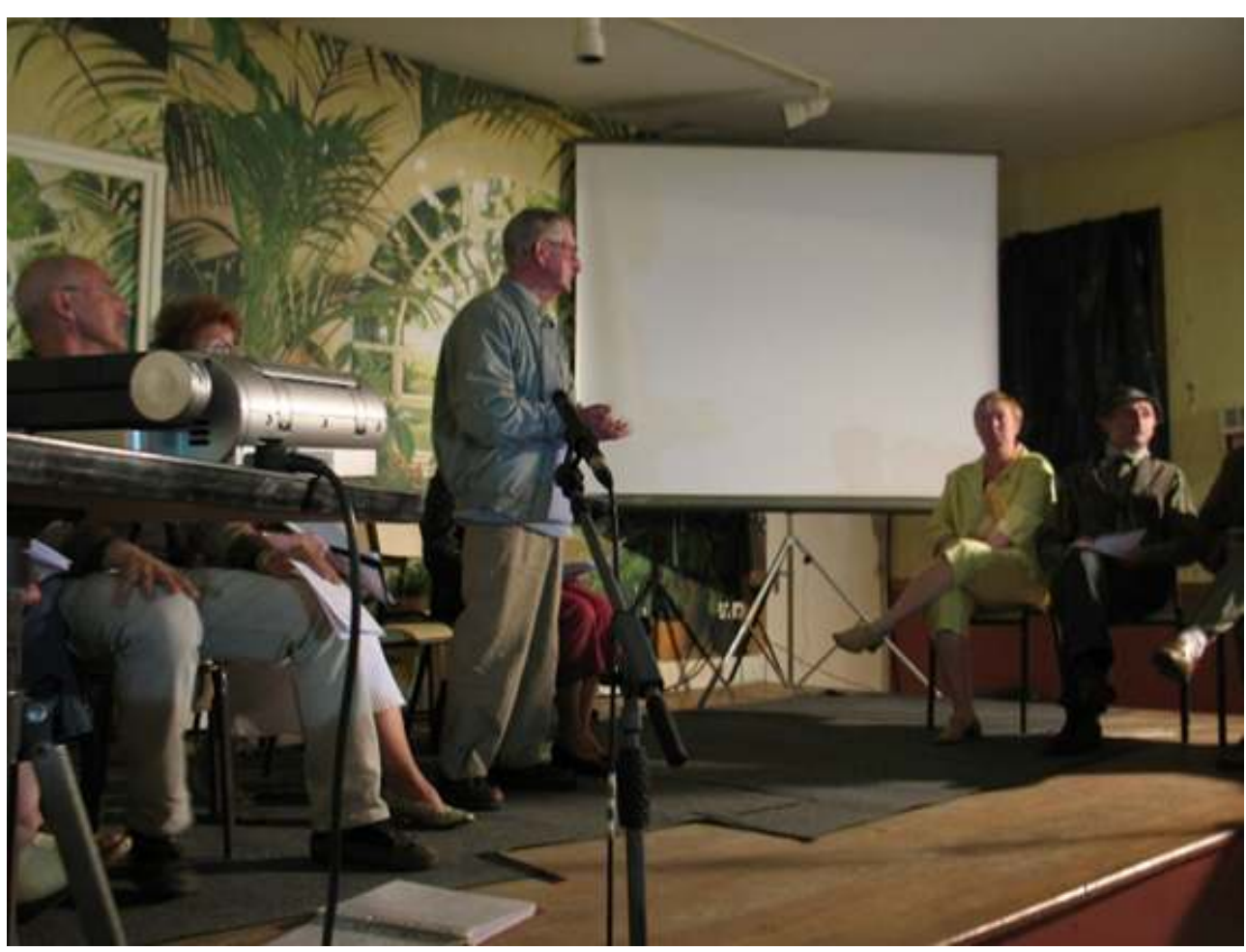

Le programme «Dans le sillon des artistes » avait prouvé qu'utiliser d'autres médias, en l'occurrence artistiques et participatifs, permettait de parler de nature et d'intéresser de nouvelles personnes. Il démontrait également que le patrimoine culturel immatériel apportait sa pierre à l'édifice dans la compréhension de l'environnement local. Il signait à la fois son apogée et la fin du programme intitulé comme tel.

\subsection{Les relations homme-nature}

Cette «méthode » s'est renouvelée. Ainsi, le recours au patrimoine culturel immatériel, plus précisément à la mémoire du territoire et des pratiques liées à la gestion d'espaces 
considérés aujourd'hui comme naturels, a pu dans certains programmes apporter une plus-value quant à la mobilisation des habitants. Et c'est pourquoi, peu à peu, les travaux ethnologiques ont repris leur place au sein des politiques du parc. Rappelons que, dans certaines parties du territoire, ces approches étaient toujours fortement employées. Les actions entreprises sur le marais audomarois aujourd'hui reconnu dans le cadre du programme «Man and Biosphere ${ }^{3}$ » de l'Unesco a servi de référence dans d'autres projets, comme pour une autre zone humide du territoire : la basse vallée de la Slack.

L'animation territoriale de la basse vallée de la Slack s'appuie, depuis 2007, sur trois volets, considérés comme dépendants les uns des autres pour un territoire équilibré :

- le volet agricole : sans l'élevage, dont l'activité se marie avec les caprices de la nature (ce marais est immergé plusieurs semaines pendant l'année), la basse vallée de la Slack ne présenterait plus ce paysage ouvert ;

- le volet hydraulique : l'omniprésence de l'eau, ses effets sur les activités humaines, sa gestion;

- le volet naturel : la basse vallée de la Slack est réputée pour être un territoire des plus intéressants du point de vue floristique et faunistique, notamment avifaunistique.

Cette animation territoriale, dont la gouvernance était assurée entre les élus, les partenaires institutionnels et les usagers directs de ce territoire (chasseurs et agriculteurs), a pu connaître des périodes plus ou moins consensuelles. C'est pourquoi, au moment d'un nouveau renouvellement du contrat d'animation, en 2015, les partenaires financiers ont réaffirmé la nécessaire approbation et implication des habitants. Les acteurs doivent donc partager un objectif commun, celui de la préservation du territoire comme bien commun. D'autant qu'à cela s'ajoute la posture du parc qui « impulse », mais doit, au bout d'un moment, se retirer au profit d'un autre coordinateur. Cette posture ne se décide pas, elle doit se préparer.

Ainsi, le parc a proposé la conduite d'une étude, sous la coordination d'Hélène Melin, maître de conférences en ethnosociologie à l'université de Lille. Il s'agissait de travailler non plus seulement sur l'histoire du lieu, mais aussi sur les perceptions qu'ont de la Slack les habitants de ce territoire. Qu'en connaissent-ils? Comment le vivent-ils? Le comprennent-ils ? L'objectif était d'éclairer certains questionnements :

- où en sont aujourd'hui les relations qu'entretiennent les habitants avec cet environnement?

- quels leviers utiliser pour les impliquer dans la préservation de ce patrimoine?

- quels axes exploiter pour que la Slack devienne aux yeux de tous un bien commun?

Ce premier travail a mis en lumière toutes les composantes du patrimoine de la Slack. Cette basse vallée n'est pas seulement un milieu, un lieu de production, elle est également un lieu d'histoire, de mémoires, et aussi de contemplation. L'étude a en outre révélé que cet aspect de la Slack était peu connu. Il a donc été proposé de valoriser ce qui le méritait, à savoir l'histoire de la Slack. Un livret de témoignages a été réalisé, illustré par un dessinateur habitant le territoire (figure 3). Puis, une action engagée avec un groupe de photographes amateurs a cherché à montrer ce territoire sous l'angle du reportage en immersion, permettant à des non-usagers de visiter la Slack de l'intérieur (figure 4). Enfin, des conférences sur l'histoire locale ainsi que des randonnées, encadrées, à plusieurs voix, ont donné à voir le marais. En utilisant des angles de connaissance différents, ce travail a pu susciter un premier intérêt chez des acteurs non usagers du territoire. Il a mis en exergue le fait que c'est la complémentarité des sujets et des points de vue relatifs à cette zone humide qui permettait de l'identifier comme un bien commun. 
Figure 3. Livret de témoignages "Vallée de la Slack. Paroles d'habitants", 2015

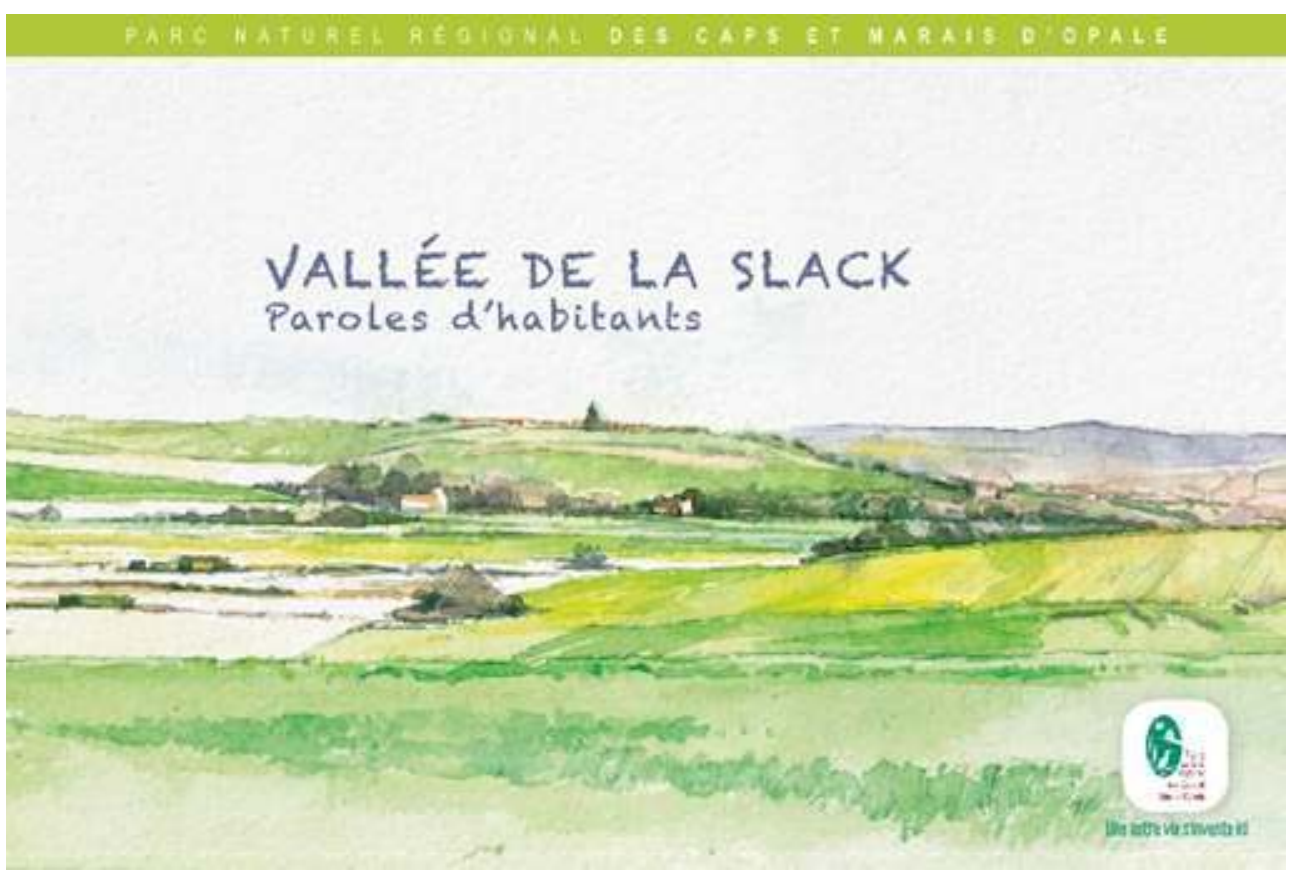

Figure 4. Un habitant impliqué dans le projet à la découverte de la vallée de la Slack , 2017

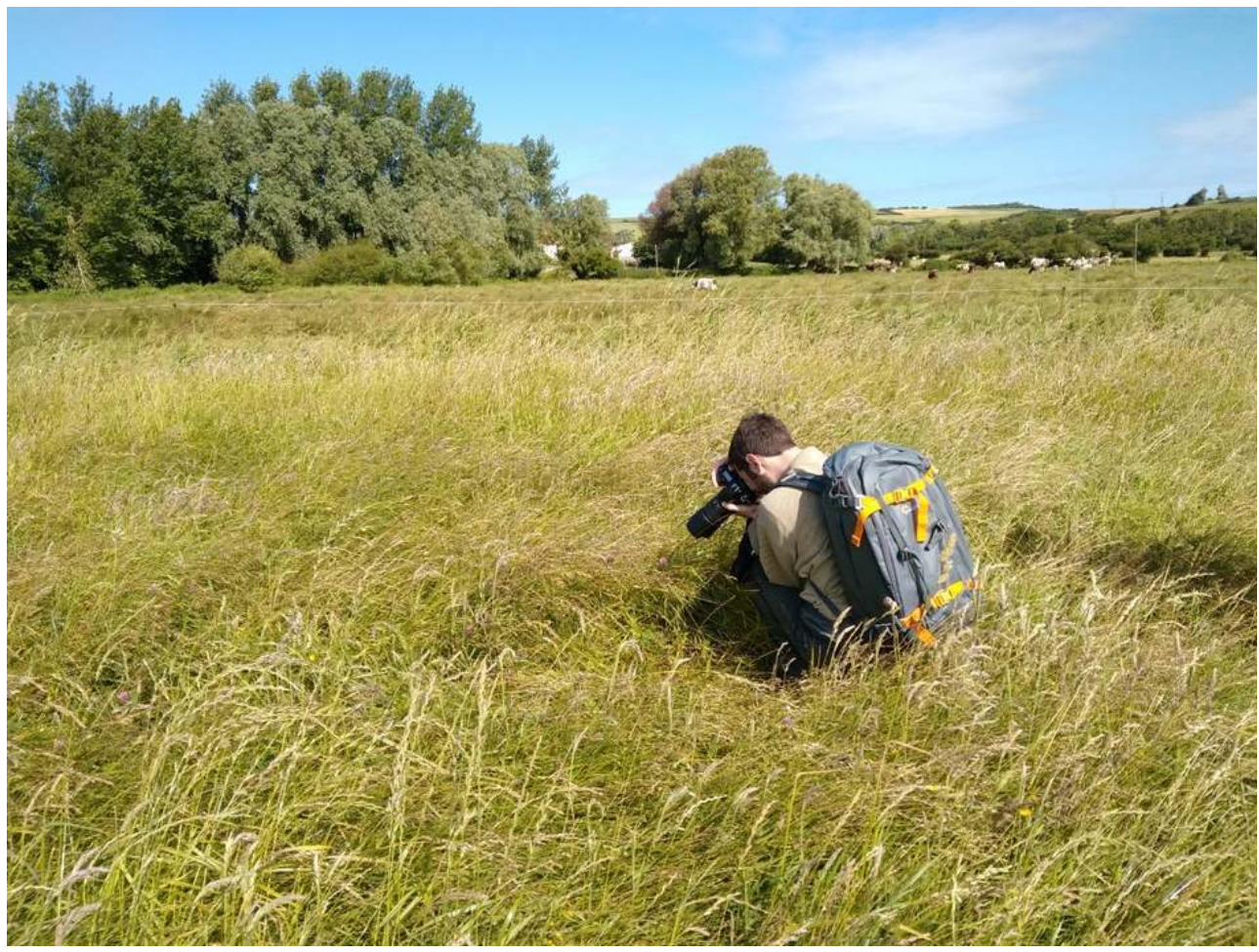

Source : PNRCMO 


\section{Mobiliser pour le bien commun ?}

\subsection{Un positionnement différent} employé pour désigner la même chose. Là où les habitants parlent de monts ou de riez, le parc évoque les pelouses calcicoles. Le marais des habitants est qualifié de zone humide par le parc. Nous sommes ici face à deux appréhensions du territoire, via une culture vernaculaire et une culture scientifique. C'est cette culture scientifique qui semble aujourd'hui être associée à l'image du parc. Cela aboutit à un hiatus, puisque le parc cherche à promouvoir un territoire accessible et compréhensible par tous; un de ses objectifs étant d'amener les habitants à s'engager.

Les enquêtes ont donc, dans certains cas, permis au parc de se repositionner comme acteur du territoire au même titre que d'autres acteurs associatifs locaux. Elles ont pu valoriser la parole des habitants et lui donner sa place au milieu de discours plus argumentés.

Elles ont également contribué à diversifier les points de vue et les regards portés sur un territoire. Les programmes d'implication des habitants mêlent dorénavant artistes, scolaires, élus, habitants, historiens, gestionnaires, etc. Certaines façons de voir le territoire pourront toucher les habitants à différents niveaux de conscience (Jung, 1964). Rappelons que l'être humain est composé de quatre sphères : le spirituel, l'émotionnel, le corporel et l'intellectuel. Ces sphères renvoient à des modes de relation que l'homme entretient avec n'importe quel élément.

Hélène Melin (2017), dans son travail de synthèse de la brochure "Les parcs et les relations homme-nature ", les résume ainsi :

- le spirituel : la nature comme un nous, l'homme est un élément de la nature ;

- l'émotionnel : la nature vue comme un lien, une émotion, un don;

- le corporel : la nature comme une possibilité, elle vient répondre à un besoin ;

- l'intellectuel : la nature comme une leçon, l'homme connaît la nature d'une manière rationnelle et scientifique.

Mêler les approches permet de toucher les habitants par l'une ou l'autre de ces sphères. Et parfois, faire appel aux émotions peut remplacer bien des discours scientifiques dans l'envie de s'engager.

\subsection{Territoire, bien commun et habitants}

Peu à peu, le parc a dirigé ses programmes d'implication des habitants en rapprochant le territoire du bien commun.

L'objectif de cette nouvelle approche est de considérer que chaque habitant peut entendre qu'il faut préserver un milieu, mais il doit être en mesure de procéder à cette analyse en partant de ses connaissances et ressentis. Ainsi, préserver un coteau de pelouses calcicoles sera nécessaire pour un naturaliste du point de vue de la biodiversité que recèlent ces milieux; évident pour un touriste qui souhaitera profiter du paysage qu'un sentier de randonnée lui dévoilera; important pour un féru d'histoire locale qui tentera de comprendre le rôle qu'a pu jouer ce lieu au cours de la guerre; riche pour un 
instituteur qui cherchera à travailler avec ses élèves sur la notion de patrimoine. Le bien commun, c'est l'accumulation de toutes ces évidences et donc de leviers d'action possibles que le parc, comme d'autres gestionnaires, pourra utiliser afin que les habitants se sentent pleinement investis dans sa préservation.

Par ailleurs, travailler de la sorte implique qu'au-delà des connaissances scientifiques, d'autres formes de connaissances, tout aussi légitimes, peuvent coexister. Il ne s'agit plus d'être en opposition, mais en complémentarité. Les exemples sont nombreux, en ce sens, nous pourrions citer ici la complémentarité de connaissances vernaculaires du berger dans son métier (quelles fleurs les moutons aiment-ils? comment régénéraient-ils le milieu ? etc.) avec celle du gestionnaire d'espaces naturels, convaincu que la qualité du milieu est une conséquence immédiate de la gestion pastorale des coteaux.

\section{Conclusion}

Que les habitants considèrent un élément de patrimoine, d'un paysage ou d'un territoire comme bien commun apporte de premiers résultats prometteurs. En effet, sur la Slack, l'opération "À la découverte de la vallée de la Slack » de l'été 2017 a montré que le croisement des regards et des envies amène à considérer le territoire de manière plus positive et à le partager davantage. C'est dans le dialogue et la démonstration que les habitants d'une part découvrent le territoire par tous ces angles et, d'autre part l'appréhendent mieux. Considérer un élément du territoire comme un bien commun ne hiérarchise aucunement les points de vue et permet à tous les habitants de s'y retrouver.

Cette nouvelle posture soulève d'autres interrogations que le parc découvre au fur et à mesure des projets qu'il développe. Ressentir une émotion pour un paysage peut servir de déclic aux habitants pour se mettre en marche. Toutefois, les actions entreprises alors pourraient ne pas être tout à fait adaptées, les habitants manquant de clés de lecture pour apporter des solutions, au regard des enjeux. Les conflits d'usage pourraient alors s'exacerber. Il faut donc veiller continuellement à ce que cette approche soit associée à une démarche pédagogique forte pour éviter ces écueils.

À l'inverse, à l'heure où l'individualisme s'exacerbe, la notion de bien commun peut être galvaudée. Les habitants pourraient considérer que si la nature est un bien commun, ils ne portent aucune responsabilité quant à son état. Si c'est commun, ce n'est pas unique, donc cela ne présente pas d'intérêt. Cette posture pourrait dévaloriser à terme le territoire.

Il convient donc de manier la notion de bien commun avec prudence pour que le résultat soit à la hauteur des espérances. L'objectif étant bien que les habitants se mobilisent pour un territoire à la qualité préservée. 


\section{BIBLIOGRAPHIE}

Carré L., 2007, « Histoire des coteaux calcaires du Pays de Licques à travers l'exploitation pastorale - À la découverte du pays des "herbes tremblantes" ».

Jung C. G., 1964, L'homme à la découverte de son âme, Albin Michel, $352 \mathrm{p}$.

Melin H., 2017, « Les relations homme-nature dans les parcs - Espaces naturels régionaux ».

Panossian D., janvier 2008, « Les habitants dans le sillon des artistes », Espaces naturels, $\mathrm{n}^{\circ} 21$, http://espaces-naturels.info/habitants-dans-sillon-artistes, consulté le 16/02/2019.

\section{NOTES}

1. Fédération des Parcs naturels régionaux. Les Parcs naturels régionaux, www.parcs-naturelsregionaux.fr (consulté le 16/02/2019).

2. Laurence Carré, ancienne ethnologue à Espaces naturels régionaux a été missionnée afin de réaliser une étude ethnosociologique sur les coteaux calcicoles.

3. L'action du Parc sur le marais audomarois est ancienne et, par conséquent, ce territoire bénéficie d'études spécifiques. L'approche ethnologique s'était jusqu'alors principalement intéressée à la pratique maraîchère (Piault Colette, Le Brouck, CNRS Audiovisuel, Les Films du quotidien (1972) http://www.film-documentaire.fr/4DACTION/w_fiche_film/32276_1; Pascale Lepers, "Le patrimoine culturel du marais de Saint-Omer », 1982, http://www.culture.gouv.fr/ content/download/44595/354595/version/1/file/Ethno_Lepers_1982_260a.pdf. Peu d'actions avaient été entreprises afin de mobiliser plus largement sur les problématiques du marais. Ainsi, une collecte de témoignages a été réalisée auprès de maraîchers, mais aussi d'élus, d'éleveurs ou de tenanciers de café. L'objectif de cette collecte était de mieux appréhender le marais dans son ensemble. Cette collecte auprès d'une vingtaine de personnes s'est traduite par :

- la réalisation d'un livret de rencontres, illustré par deux dessinatrices ;

- en parallèle et, dans le même état d'esprit, un spectacle contemporain, participatif.

Peu à peu, le marais s'est ouvert à des publics moins avertis. Il va de pair avec la recherche de l'obtention de la qualification Man and Biosphere sur le marais audomarois. Ce label de l'Unesco sanctionne les territoires où les pratiques de l'homme influent fortement sur la qualité du milieu naturel.

\section{RÉSUMÉS}

Le Parc naturel régional des Caps et Marais d'Opale, territoire à dominante rurale, situé dans les Hauts-de-France, est constitué d'un environnement fortement anthropisé, moins bien appréhendé par ses habitants, peu sensibilisés à ses valeurs ou son histoire. C'est pourquoi le Syndicat mixte du parc a développé un programme de mobilisation des habitants par la pratique 
artistique. L'objectif était d'amener les habitants à se rencontrer autour d'une création collective participative. Ces actions collectives ont peu à peu servi de supports pour transmettre un patrimoine, une connaissance, une ou des histoires centrées sur le territoire. Justifié par une mobilisation des habitants suffisamment conséquente, le Syndicat mixte du parc a cherché à travailler sur des projets confrontant les regards, associant les différentes thématiques, bref, transcendant les frontières pour montrer que l'éducation au territoire, c'est l'éducation au bien commun. Ce postulat permet d'associer des habitants d'horizons divers dans un objectif partagé.

The Caps et Marais d'Opale Regional Nature Park, a rural space located in the Hauts-de-France area, mainly consists in an anthropic environment less known from the inhabitants. Those are less aware of the values or the history of the territory. That's why the RNP syndicat mixte developed a program whom objective was to mobilize the inhabitants through artistic practical. The aim was to realise a collective creation with some inhabitants. Those actions have gradually served to transmit the heritage, the knowledge or one or some stories about the territory. Thanks to the audience of those projects, the RNP decided to work on some projects crossing over thematics, viewpoints, getting out of the bundaries. In consequence, those programs show that education to a space is not so far from education to a commons. That idea allows to associate inhabitants coming from different origins in a shared objective.

\section{INDEX}

Mots-clés : héritage, nature, pratiques, transmission, culture, Parcs naturels régionaux

Keywords : heritage, nature, practises, passing on, culture, regional nature Park

\section{AUTEUR}

\section{DELPHINE PANOSSIAN}

Au sein de la mission Patrimoine culturel du PNR Caps et Marais d'Opale, Delphine Panossian a mis en œuvre des actions de médiation artistique et sur la transmission du patrimoine culturel. Son travail porte actuellement sur la transition énergétique.

dpanossian@parc-opale.fr 